\title{
Modeling waveguides in photonic woodpiles using the fictitious source superposition method
}

\author{
Dougal J. Kan,* Ara A. Asatryan, Christopher G. Poulton, Kokou B. Dossou, and Lindsay C. Botten \\ Centre for Ultrahigh-bandwidth Devices for Optical Systems (CUDOS) and Department of Mathematical Sciences, \\ University of Technology, Sydney, P.O. Box 123, Broadway, New South Wales, 2007, Australia. \\ ${ }^{*}$ Corresponding author: Dougal.J.Kan@student.uts.edu.au
}

Received October 25, 2010; revised January 25, 2011; accepted January 26, 2011; posted January 26, 2011 (Doc. ID 137133); published March 17, 2011

\begin{abstract}
We extend the fictitious source superposition method in order to model linear defects in photonic woodpiles, and we use the method to model a waveguide that is created by changing either the radius or refractive index of a single rod of an infinite woodpile composed of chalcogenide glass cylinders. In one instance, a nearly constant dispersion was observed over a sizable $k_{x}$ interval, where $k_{x}$ is the Bloch vector in the waveguiding direction, making this a compelling geometry for slow-light waveguides. The principal advantage of the method is that it does not rely on a supercell, thus avoiding what is possibly the greatest source of inefficiency present in most of the other methods that are used for modeling these structures. Instead, the method proceeds by placing an artificial source inside each rod of the defect layer and then subsequently taking an appropriate field superposition to remove all but one of these sources. The remaining source can then be used to mimic the fields that would be produced by a defect rod. 두 2011 Optical Society of America
\end{abstract} OCIS codes: $\quad 260.0260,260.2110,050.0050,050.1960$

\section{INTRODUCTION}

A photonic crystal's (PC) ability to precisely manipulate light stems from the existence of photonic bandgaps, that is, frequency ranges over which the propagation of light along specific directions is forbidden within the crystal. These bandgaps, in turn, result from Bragg scattering due to the periodic variations of the material index [1]. Thus, a PC whose refractive index varies periodically in three dimensions (3D), such as a photonic woodpile [2-6] (see Fig. 1), can potentially suppress light propagation in all directions, irrespective of polarization (known as a complete bandgap). Introducing a defect by locally destroying the periodicity of the PC can create defect modes that have frequencies lying within a bandgap, where the fields of such modes are necessarily bound to the defect, being unable to propagate far into the bulk crystal, where the bandgap remains intact. This mechanism serves as a basis for constructing photonic-crystal waveguides, where the patterning of the PC must be sufficiently accurate in order to minimize the amount of light that is scattered in directions not confined by the bandgap. An area of great interest recently, and where the elimination of parasitic scattering is critical, is the design of "slow-light" waveguides, whereby the dispersion of the waveguide is engineered so that light entering the waveguide is slowed down dramatically, leading to a host of compelling applications [7]. In this regime, extrinsic losses become catastrophic [조, 9 ], because the light has more time to interact with the walls of the PC. For well-engineered 3D PCs, scattering losses arising from extrinsic factors, such as surface roughness, can be prevented provided that the bandgap is complete, and, hence, lossless waveguiding might be achievable if losses arising from material absorption are kept small.

In this paper we describe how the fictitious source superposition (FSS) method can be applied to the problem of modeling a cylindrical defect contained in a woodpile cladding composed of infinitely long cylindrical scatterers (defect structures incorporated into woodpiles and operating at wavelengths near $1.55 \mu \mathrm{m}$ have already been successfully realized using the wafer bonding technique [10], including woodpile waveguides $[11,12])$. In an earlier paper we described how a semianalytic method, known as the multipole method, could be extended to accommodate defect rods within a finite woodpile cladding [13]. While that approach proved to be an efficient way of modeling a CROW layer embedded in a host woodpile, generating the dispersion curves for a linear waveguide remained difficult because of the narrow linewidth of the spectral features. Further, the method required a moderately large supercell in the grating direction, and this offset the benefits of expressing the electromagnetic field identities in a natural basis for the problem. Indeed, every numerical study of intentional defects in woodpiles, so far, has relied heavily on a supercell approach (see, e.g., [14]), whereby periodic boundary conditions are imposed at an arbitrary distance from the defect center. Specifically, the plane wave expansion [15] (PWE) and finite-difference time-domain [16] (FDTD) methods are used almost exclusively in the study of these structures $[11,12,17-20]$ (typically, the PWE method is used to calculate the eigenmodes of the infinite stack, while FDTD modeling is used to locate resonances of the truncated stack and also to compute the temporal response of the woodpile to an input beam). For both of these methods, the supercell is used to artificially reduce the modeled domain to a size that can be computed within a reasonable time. However, increasing the size of the supercell, thereby making the structure more similar to the physical structure being modeled, leads to rapidly increasing demands on computing time and memory.

In earlier work, we developed FSS methods, which do not rely on a supercell, to model modes that are highly extended, 


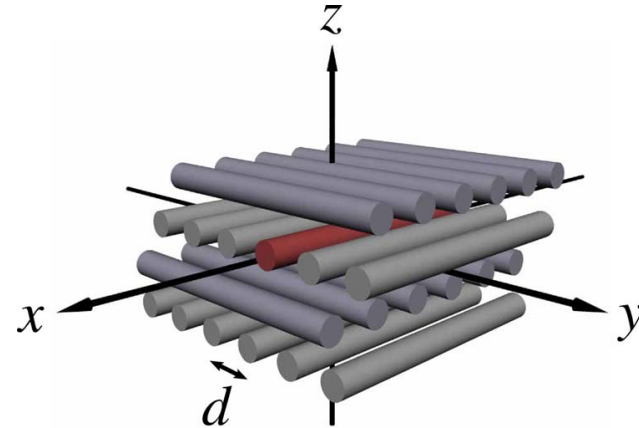

Fig. 1. (Color online) Photonic woodpile: a linear waveguide can be created by changing the properties of a single cylinder (red).

such as that happens when near the cutoff $[21,22]$. In this paper, we generalize those techniques in order to avoid the computational overhead associated with a supercell. The FSS method instead relies on the placement of sources inside each cylinder of the waveguide layer and then takes an appropriate superposition of fields to mimic a finite number of defects in an infinite array. This can be done efficiently because the multipole formulation lets one manipulate the outgoing fields directly; however, in doing so, one forgoes the ability to deal with a finite number of layers. This is a new method capable of producing accurate results when the fields of the defect mode extend great distances into the 3D structure. The substantial performance gains realized by the FSS method meant that it was possible to obtain well-resolved dispersion curves for the linear waveguide, and also it allowed us to optimize the waveguide geometry for operation in the slow-light regime, which occurs when the group velocity, $\mathbf{v} \stackrel{\text { def }}{=} \nabla_{\mathbf{k}} \omega$, along the waveguiding direction is small, that is, when $v_{x}=\partial \omega / \partial k_{x}$ $\ll c$, where $x$ is the direction parallel to the linear waveguide, $\omega$ is the angular frequency, $\mathbf{k}$ is the Bloch vector, and $c$ is the speed of light in a vacuum. In one instance, we observed that the slow-light condition was satisfied over a continuous interval spanning $\sim 15 \%$ of the $k_{x}$ space.

In Section 2 it is shown how the FSS formulation of [ $\underline{21}, \underline{22}]$, originally developed for $2 \mathrm{D}$ grating arrays, can be adapted to model linear defects in 3D woodpiles; the principal difference being that there is now a set of grating diffraction orders associated with the $x$ direction in addition to the usual ones associated with the $y$ direction (see [23] for a list of references to early work on the use of fictitious sources in the study of $2 \mathrm{D}$ systems of diffracting rods). The multipole theory for woodpiles is lengthy and has appeared previously in [24,13]. In this paper we have only included the parts of the multipole theory that are of immediate relevance, and, for brevity, mathematical descriptions of many of the terms appearing in the multipole expressions have been omitted. However, where necessary, the notation has been kept consistent with that of [13], which should be consulted if the precise definitions are required, and the appropriate equation numbers of [13] are referenced in the theory that follows. The measures that were taken to test the correctness of our code are outlined in Section 3 , along with implementation notes. Results were obtained for a chalcogenide woodpile waveguide, where the defect size and refractive index were allowed to vary in turn, and are presented in Section $\underline{4}$.

\section{THEORY}

\section{A. Overview}

The theory in this section is organized as follows. In Section 2.B, multipole expressions for the fields produced by a single grating are given, where a fictitious source, $\mathbf{Q}_{j}$, is embedded inside each cylinder of the grating. All of the multipole grating field identities remain the same as those in $[24,13]$, except possibly for the appearance of an additional term representing the contribution of $\mathbf{Q}_{j}$ to the fields. In particular, it will become apparent that the presence of fictitious sources does not affect the expressions for the grating reflection and transmission matrices ( $\mathbf{R}$ and $\mathbf{T}$ ), which relate the diffracted fields to the incoming fields.

Having obtained the modified grating expressions, the outgoing fields emanating from the grating are then expressed purely in terms of the fictitious source coefficients. The fictitious sources may then be chosen so as to mimic a cylinder having a different size and refractive index from the original one, in effect creating a defect. However, using the fictitious sources to manipulate the outgoing fields of each cylinder independently from those of the other cylinders in the grating is usually impractical, if not impossible. To this end, the problem is formulated so that the sources are quasiperiodic. Preserving the quasiperiodicity of the fields in this way also means that $\mathbf{R}$ and $\mathbf{T}$, along with the reflection matrix of the semi-infinite cladding [24], $\mathbf{R}_{\infty}$, can be brought to bear on the problem of expressing the outgoing fields in terms of $\mathbf{Q}_{j}$.

This simplification leads to a conceptually straightforward procedure for modeling a woodpile that contains a single defect rod: one simply integrates the modified fields over the quasiperiodic phase \{the idea of integrating over the Brillouin zone (BZ) in order to compute the defect modes of PCs is also employed in the resolvent approach [25]\}. For this to work, further constraints must be imposed on $\mathbf{Q}_{j}$ so that only those sources contained in cylinders of our choosing will remain unchanged by the superposition process, while all other sources disappear. It happens that averaging the fields in this way amounts to averaging the multipole field coefficients and, consequently, the solution of the superposed problem is trivially related to the solutions of the unsuperposed problems. The details of the superposition step are given in Subsection 2.C.

\section{B. Fictitious Source Superposition Grating Field Identities}

We first consider a single grating that consists of uniform cylinders that have a refractive index of $n_{c}$ and are embedded in a background medium of refractive index $n_{b}$ (it is assumed that the cylinders and background region are both isotropic). Suppose an incoming plane wave, specified by the wave vector $\mathbf{k}=\left(\alpha_{0}, \beta_{0},-\gamma_{0}\right)$, is incident from above the grating, so that the components of $\mathbf{k}$, in spherical coordinates, are $\alpha_{0}=k n_{b} \sin \varphi \cos \phi, \beta_{0}=k n_{b} \sin \varphi \sin \phi$ and $\gamma_{0}=k n_{b} \cos \varphi$, where the free-space wavenumber and wavelength are $k=$ $2 \pi / \lambda$ and $\lambda$, respectively, and where $\varphi$ and $\phi$ are the conical angles [see Fig. 2(a)].

A grating that is aligned parallel to the $x$ axis, as in Fig. 2(b), generates diffracted plane waves having a $k_{y}$ component of $\beta_{q}$, while a $y$-aligned grating generates plane waves having a $k_{x}$ component of $\alpha_{p}$. Here, $p$ and $q$ are integers used to label the usual grating diffraction orders (or "channels"): $\alpha_{p}=\alpha_{0}+$ $2 \pi p / d_{x}$ and $\beta_{q}=\beta_{0}+2 \pi q / d_{y}$, where $d_{x}\left(d_{y}\right)$ is the pitch of any 


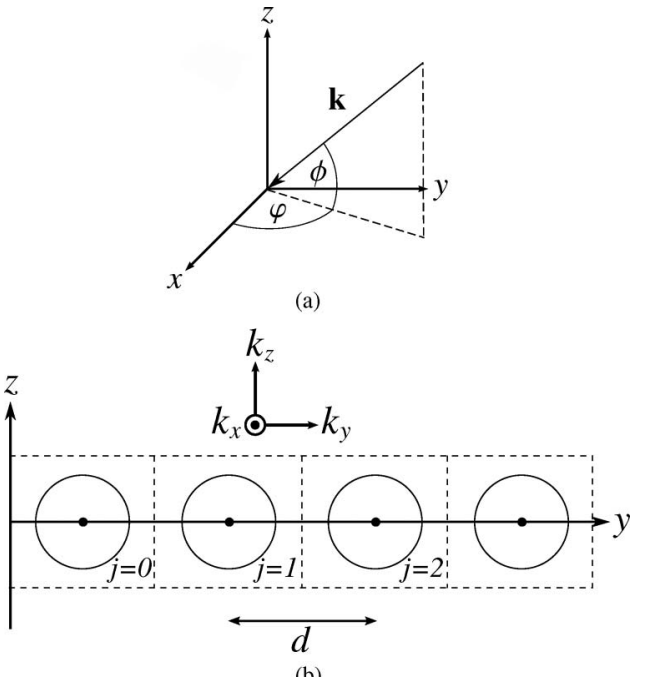

(b)
In order to use the multipole method to determine the fields inside the grating, $p$ must first be fixed; in effect projecting the problem onto the $y z$ plane [24]. The multipole method is also used to determine the diffracted fields $\boldsymbol{f}_{D, p}^{ \pm}$, where $p$ is used to denote the part of the field having an $x$ dependency of $\exp \left(i \alpha_{p} x\right)$, that is,

$$
\boldsymbol{f}_{D, p}^{ \pm}=\left[\begin{array}{l}
{\left[f_{D,(p, q)}^{\mathrm{TE} \pm}\right]} \\
{\left[f_{D,(p, q)}^{\mathrm{TM}}\right]}
\end{array}\right]
$$

in which $(p, q)=\sigma^{-1}(s)$ and $q=\ldots,-1,0,1, \ldots$. In the vicinity of the cylinders, it is valid to expand the longitudinal components of the fields, i.e., $E_{x}$ (electric) and $H_{x}$ (magnetic), in terms of cylindrical harmonics. Suppose $\mathbf{r}=(\rho, \theta, x)$ is a point (expressed in cylindrical coordinates) exterior to cylinder $j$. Whether or not fictitious sources are present, the fields at $\mathbf{r}$ are

$$
V_{p, x}(\mathbf{r})=\sum_{n=-\infty}^{\infty}\left[A_{p, n}^{j, V} J_{n}\left(k_{e \perp} \rho\right)+B_{p, n}^{j, V} H_{n}\left(k_{e \perp} \rho\right)\right] e^{i n \theta} e^{i \alpha_{p} x},
$$

Fig. 2. (a) Spherical coordinates of the wave vector $\mathbf{k}=\left(k_{x}, k_{y}, k_{z}\right)$.
(b) Successive unit cells of a grating whose cylinders are parallel to the $x$ axis, with $j$ indexing the cylinders in order of increasing displacement along the $y$ axis.

grating aligned parallel to the $y$ axis ( $x$ axis), although it is typical to use a common pitch $d=d_{x}=d_{y}$, which we do here for notational simplicity. A pair of integers $(p, q)$ can thus be used to specify a single woodpile diffraction order, where it is understood that this represents a plane wave having $x$ and $y$ dependencies of $\exp \left(i \alpha_{p} x\right)$ and $\exp \left(i \beta_{q} y\right)$, respectively. A bijective map $\sigma: \mathbb{Z} \times \mathbb{Z} \rightarrow \mathbb{Z}$ is required so that each diffraction order $(p, q) \in \mathbb{Z}^{2}$ can be uniquely identified using an index $s \in \mathbb{Z}$; in other words, $s=\boldsymbol{\sigma}(p, q)$ and $(p, q)=\boldsymbol{\sigma}^{-1}(s)$. Each order consists of an upward and a downward traveling wave, i.e., having a $z$ dependency of $\exp \left(i \gamma_{s} z\right)$ and $\exp \left(-i \gamma_{s} z\right)$, respectively, where $\gamma_{s}^{2}=\left(k n_{b}\right)^{2}-\left(\alpha_{p}^{2}+\beta_{q}^{2}\right)$.

The theory in this section applies to gratings of either orientation; however, in order to simplify the exposition, it is assumed that the cylinders comprising the grating are aligned parallel to the $x$ axis. If, instead, the cylinders run parallel to the $y$ axis, then one simply uses a rotated coordinate system $\left(x^{\prime}, y^{\prime}, z^{\prime}\right)$, with $x^{\prime}=y, y^{\prime}=-x, z^{\prime}=z^{\prime}$. In this case, the grating diffraction orders are given by $\alpha_{p}^{\prime}$ and $\beta_{q}^{\prime}$, with the correspondences $\alpha_{p}^{\prime}=\beta_{p}$ and $\beta_{q}^{\prime}=-\alpha_{-q}$. Consequently, $\gamma_{(p, q)}^{\prime}=\gamma_{(-q, p)}$, which means that channel $(p, q)$ of the rotated system corresponds to channel $(-q, p)$ of the unrotated system. The map $\sigma^{\prime}: \mathbb{Z} \times \mathbb{Z} \rightarrow \mathbb{Z}$ associated with the rotated system must be chosen so that the channel index " $s$ " does not depend on the alignment of the grating; that is, so that $s=\sigma^{\prime}(p, q)=\sigma(-q, p)$.

It is natural to express the fields impinging on any layer as superpositions of upward and downward woodpile diffraction orders, although we have yet to take into account the fact that, in general, the electric and magnetic problems are coupled. The most appropriate way to deal with this is to further resolve each order into TE and TM polarized components. The fields can thus be represented succinctly as column vectors of the form

$$
\boldsymbol{f}^{ \pm}=\left[\begin{array}{l}
{\left[f_{s}^{\mathrm{TE} \pm}\right]} \\
{\left[f_{s}^{\mathrm{TM} \pm}\right]}
\end{array}\right]
$$

where + and - denote fields propagating upward and downward, respectively, and where $f_{s}^{\mathrm{TE}}$ is the TE component of the $s$ th order, and $f_{s}^{\mathrm{TM}}$ is the TM component [13]. with $V=E$ and $V=H$ in turn, where $k_{e \perp}=\left[\left(k n_{b}\right)^{2}-\alpha_{p}^{2}\right]^{1 / 2}$ is the transverse wavenumber of the exterior fields, and $J_{n}$ and $H_{n}$ are Bessel and Hankel functions of the first kind, respectively. Equation (3) has a simple physical interpretation, with the Bessel functions representing the incoming fields and the Hankel functions representing the outgoing fields sourced on the boundary of cylinder $j$. When there are no fictitious sources, the multipole coefficients are quasiperiodic, so that $A_{p, n}^{j, V}=A_{p, n}^{0, V} \exp \left(i \beta_{0} j d\right)$ and $B_{p, n}^{j, V}=B_{p, n}^{0, V} \exp \left(i \beta_{0} j d\right)$. In this case, the fields inside cylinder $j$ are simply

$$
V_{p, x}(\mathbf{r})=\sum_{n=-\infty}^{\infty} C_{p, n}^{j, V} J_{n}\left(k_{i \perp} \rho\right) e^{i n \theta} e^{i \alpha_{p} x},
$$

where $k_{i \perp}=\left[\left(k n_{c}\right)^{2}-\alpha_{p}^{2}\right]^{1 / 2}$ is the transverse wavenumber of the interior fields, and $C_{p, n}^{j, V}=C_{p, n}^{0, V} \exp \left(i \beta_{0} j d\right)$.

We may assume, without loss of generality, that $j=0$. The incoming fields $\left(A_{n}^{0, V}\right)$ and the outgoing fields $\left(B_{n}^{0, V}\right)$ are related through the Rayleigh identity

$$
\mathcal{A}_{p}=\mathcal{S}_{p} \mathcal{B}_{p}+\mathcal{J}_{p}^{-} \mathbf{Z} \mathbf{X}_{p}^{-} \boldsymbol{f}_{I, p}^{-}+\mathcal{J}_{p}^{+} \mathbf{Z} \mathbf{X}_{p}^{+} \boldsymbol{f}_{I, p}^{+},
$$

with $\mathcal{A}_{p}=\left[\left[A_{p, n}^{0, E}\right]^{T} \quad\left[A_{p, n}^{0, H}\right]^{T}\right]^{T}, \mathcal{B}_{p}=\left[\left[B_{p, n}^{0, E}\right]^{T} \quad\left[B_{p, n}^{0, H}\right]^{T}\right]^{T}$, where the superscript $T$ denotes the matrix transpose and the row index $n$ ranges over all multipole orders (hence, $\mathcal{A}_{p}$ and $\mathcal{B}_{p}$ are column vectors). Here, $\mathcal{J}_{p}^{ \pm} \mathbf{Z} \mathbf{X}_{p}^{ \pm}$converts the incoming plane wave fields, $\boldsymbol{f}_{I, p}^{ \pm}$, into cylindrical harmonics \{see Eq. (12) and Appendix A of [13]], while $\mathcal{S}_{p} \mathcal{B}_{p}$ accounts for the contribution to the incoming fields due to the outgoing fields sourced on the cylinder and each of its periodic replicates in the layer. The expressions for the entries of $\mathcal{S}_{p}$ are given by Eqs. (A3) and (A4) of [13].

By requiring that the tangential components $E_{\theta}, H_{\theta}, E_{x}$, and $E_{x}$ be continuous across the cylinder boundaries, a second equation relating $\mathcal{A}_{p}$ to $\mathcal{B}_{p}$ is obtained

$$
\mathcal{A}_{p}=-\mathcal{M}_{p} \mathcal{B}_{p}
$$

where $\mathcal{M}_{p}$ completely encapsulates the material properties (i.e., refractive index and size of the cylinders); see, e.g., 
Appendix B of [13]. Equations ()ㅡㄹ and (6) allow $\mathcal{B}_{p}$ to be expressed purely in terms of the incoming fields

$$
\mathcal{B}_{p}=-\mathcal{L}_{p} \mathcal{J}_{p}^{-} \mathbf{Z} \mathbf{X}_{p}^{-} \boldsymbol{f}_{I, p}^{-}-\mathcal{L}_{p} \mathcal{J}_{p}^{+} \mathbf{Z} \mathbf{X}_{p}^{+} \boldsymbol{f}_{I, p}^{+},
$$

where $\mathcal{L}_{p}=\left(\mathcal{M}_{p}+\mathcal{S}_{p}\right)^{-1}$ is the multipole scattering operator.

Naturally, the diffracted plane wave fields incident on the grating, $f_{D, p}^{ \pm}$, may be expressed in terms of the incoming plane waves and the outgoing multipole fields. Specifically, it can be shown that

$$
\begin{aligned}
& \boldsymbol{f}_{D, p}^{-}=\boldsymbol{f}_{I, p}^{-}+(2 / d) \mathcal{G}_{p}\left(\mathbf{Z} \mathbf{X}_{p}^{-}\right)^{-1} \mathcal{K}_{p}^{-} \mathcal{B}_{p}, \\
& \boldsymbol{f}_{D, p}^{+}=\boldsymbol{f}_{I, p}^{+}+(2 / d) \mathcal{G}_{p}\left(\mathbf{Z} \mathbf{X}_{p}^{+}\right)^{-1} \mathcal{K}_{p}^{+} \mathcal{B}_{p},
\end{aligned}
$$

where $\left(\mathbf{Z X}_{p}^{ \pm}\right)^{-1} \mathcal{K}_{p}^{ \pm}$converts the multipole fields back into plane waves \{see Eq. (17) and Appendix B of [13]\} and $\mathcal{G}_{p}$ is a diagonal matrix that is introduced to normalize energy calculations

$$
\mathcal{G}_{p}=\left[\begin{array}{cc}
\mathfrak{G}_{p} & \mathbf{0} \\
\mathbf{0} & \mathfrak{G}_{p}
\end{array}\right],
$$

$\varsigma_{p}=\operatorname{diag}\left[1 / \gamma_{s}\right]$, and $s=\sigma(p, q)$ with $p$ fixed, see Eqs. (31) and (47) of [24]. The derivation of the normalization term, $\widehat{G}_{p}$, is not given here as it is quite lengthy and requires some familiarity with the energy conservation properties of the multipole method (these properties are discussed in detail in Subsection 3.A of [26]). Substituting the multipole fields of Eq. (7) into the two equations above gives the explicit relationships between the incoming and diffracted fields incident on the grating

$$
\begin{gathered}
f_{D, p}^{-}=\mathcal{T}_{p, a} f_{I, p}^{-}+\mathcal{R}_{p, b} f_{I, p}^{+}, \\
f_{D, p}^{+}=\mathcal{R}_{p, a} f_{I, p}^{-}+\mathcal{T}_{p, b} f_{I, p}^{+},
\end{gathered}
$$

where $\mathcal{R}_{p}$ and $\mathcal{T}_{p}$ are the reflection and transmission matrices associated with the fields incoming from above (subscript $a$ ) and below (subscript $b$ ) the grating [see Eqs. ( $\underline{\mathrm{B} 1})$ and ($\underline{\mathrm{B}})$ of Appendix B].

When fictitious sources are placed inside each cylinder, the fields inside cylinder $j$ are instead

$$
V_{p, x}(\mathbf{r})=\sum_{n=-\infty}^{\infty}\left[C_{p, n}^{j, V} J_{n}\left(k_{i \perp} \rho\right)+Q_{p, n}^{j, V} H_{n}\left(k_{i \perp} \rho\right)\right] e^{i n \theta} e^{i \alpha_{p} x},
$$

see Eq. (4), where $Q_{p, n}^{j, V}$ are the fictitious source coefficients. Further, the sources are chosen to be quasiperiodic, i.e., $Q_{p, n}^{j, V}=Q_{p, n}^{0, V} \exp \left(i \beta_{0} j d\right)$. This ensures that the exterior fields retain their quasiperiodicity, and, hence, the form of the Rayleigh identity, Eq. (ㅁ), does not change. Equation (ㅁ), and thus Eq. (7), gain an additional term involving $\mathcal{Q}_{p}=$ $\left[\left[Q_{p, n}^{0, E}\right]^{T} \quad\left[Q_{p, n}^{0, H}\right]^{T}\right]^{T}$, so that instead

$$
\begin{gathered}
\mathcal{A}_{p}=-\left(\mathcal{M}_{p} \mathcal{B}_{p}+\mathcal{N}_{p} \mathcal{Q}_{p}\right), \\
\mathcal{B}_{p}=\mathcal{Y}_{p}^{-} \boldsymbol{f}_{I, p}^{-}+\mathcal{Y}_{p}^{+} \boldsymbol{f}_{I, p}^{+}+\mathcal{Y}_{p}^{\text {fs }} \mathcal{Q}_{p},
\end{gathered}
$$

where $\mathcal{Y}_{p}^{\mathrm{fs}}=-\mathcal{L}_{p} \mathcal{N}_{p}$ and, for convenience, we define $\mathcal{Y}_{p}^{ \pm}=$ $-\mathcal{L}_{p} \mathcal{J}_{p}^{ \pm} \mathbf{Z X} \mathbf{X}_{p}^{ \pm}$(expressions for $\mathcal{N}_{p}$ are given in Appendix $\underline{\mathrm{A}}$ ). Equations (8) and (9) remain valid, with $\mathcal{B}_{p}$ now given by Eq. (15), thus, Eqs. (11) and (12) can be generalized to

$$
\boldsymbol{f}_{D, p}^{-}=\mathcal{T}_{p, a} \boldsymbol{f}_{I, p}^{-}+\mathcal{R}_{p, b} \boldsymbol{f}_{I, p}^{+}+\mathcal{Q}_{p}^{-} \mathcal{Q}_{p}
$$

$$
f_{D, p}^{+}=\mathcal{R}_{p, a} f_{I, p}^{-}+\mathcal{T}_{p, b} f_{I, p}^{+}+\mathcal{Q}_{p}^{+} \mathcal{Q}_{p}
$$

with $\mathcal{Q}_{p}^{ \pm}=-(2 / d)\left(\mathbf{Z} \mathbf{X}_{p}^{ \pm}\right)^{-1} \mathcal{K}_{p}^{ \pm} \mathcal{L}_{p} \mathcal{N}_{p}$.

In order to (uniformly) change the material parameters of the cylinders of the grating when there are no fictitious sources, we need only change the boundary condition matrix $\mathcal{M}_{p}$ appearing in Eq. (6). Hence, if $\hat{\mathcal{M}}_{p}$ is the matrix for the new parameters, Eq. (므) becomes

$$
\mathcal{A}_{p}=-\hat{\mathcal{M}}_{p} \mathcal{B}_{p}
$$

Evidently, if we instead wish to use the fictitious sources to mimic these new parameters, then $\mathcal{Q}_{p}$ must be chosen in a way so as to satisfy both Eqs. (14) and (18). This leads to the first of two main relationships between the outgoing fields, i.e., $\mathcal{B}_{p}$, and the sources $\mathcal{Q}_{p}$

$$
\mathcal{B}_{p}=\mathcal{H}_{p} \mathcal{Q}_{p}
$$

where

$$
\mathcal{H}_{p}=\left(\hat{\mathcal{M}}_{p}-\mathcal{M}_{p}\right)^{-1} \mathcal{N}_{p} \mathcal{Q}_{p} .
$$

Coupling of the fields between adjacent layers of the woodpile occurs via $f_{I, p}^{ \pm}$and $f_{D, p}^{ \pm}$, i.e., via Eqs. (11) and (12), for cladding layers, and Eqs. (16) and (17), for gratings containing fictitious sources (see Fig. 3). Further, mixing occurs between different channels, so that the incoming fields $\boldsymbol{f}_{I, p}^{ \pm}$result from scattered fields produced by all other layers, where these scattered fields are radiating in all channels, rather than just in channel $p$ (this must be so, as channel $p$ for $x$-aligned gratings is physically different from channel $p$ for $y$-aligned gratings). The field equations for different values of $p$ are thus coupled and must be solved simultaneously. All of the expressions

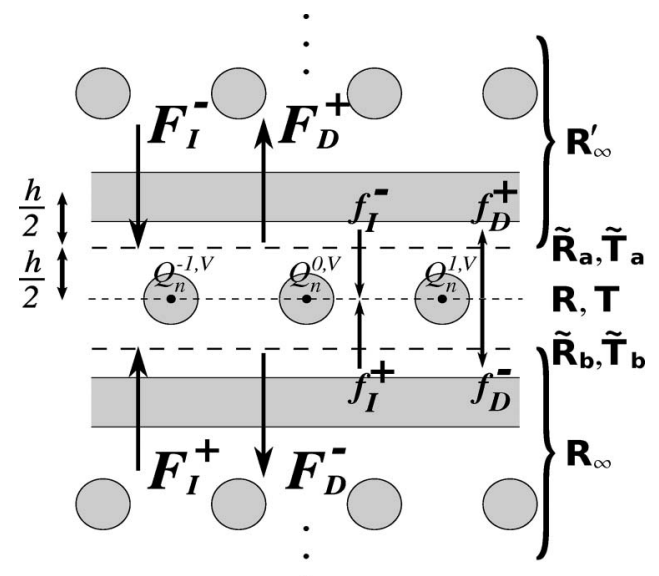

Fig. 3. The incoming $\left(\boldsymbol{f}_{I}^{ \pm}\right)$and diffracted fields $\left(\boldsymbol{f}_{D}^{ \pm}\right)$, as well as the reflection $(\mathbf{R})$ and transmission $(\mathbf{T})$ matrices associated with the waveguide layer. Each cylinder of the layer contains a fictitious source $\left(Q_{n}^{j, V}\right)$. The phases of $\boldsymbol{f}^{ \pm}$must be adjusted in order to give the waveguide layer an artificial thickness $h$ equal to the distance between adjacent layers. The phase-adjusted fields are given by $\boldsymbol{F}_{I}^{ \pm}$and $\boldsymbol{F}_{D}^{ \pm}$. This procedure also necessitates an additional pair of scattering matrices, so that there are two such matrices associated with the top surface of the waveguide ( $\tilde{\mathbf{R}}_{a}$ and $\tilde{\mathbf{T}}_{a}$ ) and another two associated with the bottom surface $\left(\tilde{\mathbf{R}}_{b}\right.$ and $\left.\tilde{\mathbf{T}}_{b}\right)$. The semi-infinite cladding regions below and above the waveguide layer are characterized by the $\mathbf{R}_{\infty}$ and $\mathbf{R}_{\infty}^{\prime}$ reflection matrices. 
derived so far can be generalized systematically to take this channel mixing into account.

Starting with Eqs. (11) and (12), it is apparent that analogous relationships must also hold between the total diffracted and incoming fields, i.e.,

$$
\begin{aligned}
& f_{D}^{-}=\mathbf{T}_{a} f_{I}^{-}+\mathbf{R}_{b} f_{I}^{+}, \\
& f_{D}^{+}=\mathbf{R}_{a} f_{I}^{-}+\mathbf{T}_{b} f_{I}^{+},
\end{aligned}
$$

where the total fields $\boldsymbol{f}^{ \pm}$have the form of Eq. (1), rather than that of Eq. (2), so that $p$ is no longer fixed, and where $\mathbf{R}$ and $\mathbf{T}$ are the general reflection and transmission matrices [see Eqs. (B3) and (B4) of Appendix B]. Similarly, Eqs. (16), (17), (15), and (19) generalize to

$$
\begin{gathered}
\boldsymbol{f}_{D}^{-}=\mathbf{T}_{a} \boldsymbol{f}_{I}^{-}+\mathbf{R}_{b} \boldsymbol{f}_{I}^{+}+\mathbf{Q}^{-} \mathbf{Q}, \\
\boldsymbol{f}_{D}^{+}=\mathbf{R}_{a} \boldsymbol{f}_{I}^{-}+\mathbf{T}_{b} \boldsymbol{f}_{I}^{+}+\mathbf{Q}^{+} \mathbf{Q}, \\
\mathbf{B}=\mathbf{Y}^{-} \boldsymbol{f}_{I}^{-}+\mathbf{Y}^{+} \boldsymbol{f}_{I}^{+}+\mathbf{Y}^{f s} \mathbf{Q}, \\
\mathbf{B}=\mathbf{H} \mathbf{Q},
\end{gathered}
$$

where

$$
\mathbf{Q}=\left[\begin{array}{l}
{\left[\mathcal{Q}_{p}^{E}\right]} \\
{\left[\mathcal{Q}_{p}^{H}\right]}
\end{array}\right],
$$

$\mathcal{Q}_{p}^{V}=\left[Q_{p, n}^{0, V}\right]$, with $\mathbf{B}$ similarly defined, and where the blocks of $\mathbf{Q}^{ \pm}, \mathbf{Y}^{ \pm}, \mathbf{Y}^{f s}$ and $\mathbf{H}$ are constructed from the blocks of $\mathcal{Q}_{p}^{ \pm}, \mathcal{Y}_{p}^{ \pm}$, $\mathcal{Y}_{p}^{f s}$, and $\mathcal{H}_{p}$, respectively, in a manner analogous to the construction of $\mathbf{R}$ and $\mathbf{T}$.

Equations (22)-(25) are the main results of this section, and they generalize the corresponding FSS expressions for 2D grating stacks reported in Subsection 2.D of [21]. These relationships, along with Eqs. (20) and (21) allow one to find the incoming fields (now the modes of an infinite structure) in Eq. (24) in terms of $\mathbf{Q}$, establishing a second linear relationship, $\overline{\mathbf{B}}=\mathbf{Z Q}$, between $\mathbf{B}$ and $\mathbf{Q}$, and thus determining $\mathbf{Q}$.

\section{Formulation for a Linear Waveguide}

The simplest defect that can be modeled using the FSS method is one where the properties of just a single cylinder of the woodpile are changed (as in Fig. 1). Fictitious sources are first placed in each cylinder of the waveguide layer, using the procedure just described. Thus, initially, the defect is one-dimensional in that the sources modify the entire row of cylinders uniformly to create a defect that is localized only in the $z$ direction. While this means that the woodpile is no longer periodic in this direction, the periodicity in the $x$ and $y$ directions is preserved. Hence, a field expansion in terms of woodpile diffraction orders is still valid, where the $k_{x}$ and $k_{y}$ components of the wave vector (i.e., $k_{x} \equiv \alpha_{0}$ and $k_{y} \equiv \beta_{0}$ ) are now used to specify a mode having wavenumber $k$, and the diffraction orders are now interpreted as being modes whose Bloch vector differ from $\left(k_{x}, k_{y}\right)$ only by a reciprocal lattice vector. The fields of the woodpile are then integrated over one dimension of the BZ, thereby leaving a fictitious source only in a single rod.

Let $k$ and the in-plane wave vector $\left(k_{x}, k_{y}\right)$ be fixed. The incoming fields must first be expressed in terms of $\mathbf{Q}$. It is necessary to apply a symmetrical padding to the waveguide layer, giving the layer an artificial thickness of $h$ equal to the distance between adjacent layers. The phase origin of the fields incident above (below) the grating must, thus, be shifted upward (downward) by an amount $h / 2$. This is achieved by replacing $\boldsymbol{f}_{I}^{ \pm}$and $\boldsymbol{f}_{D}^{ \pm}$in Eqs. (2) and (르) with

$$
\boldsymbol{F}_{I}^{ \pm}=\mathbf{P}^{-1} \boldsymbol{f}_{I}^{ \pm}, \quad \boldsymbol{F}_{D}^{ \pm}=\mathbf{P} \boldsymbol{f}_{D}^{ \pm},
$$

respectively, where $\mathbf{P}$ is a diagonal matrix such that

$$
\mathbf{P}=\left[\begin{array}{ll}
\mathcal{P} & 0 \\
0 & \mathcal{P}
\end{array}\right],
$$

with $\mathcal{P}=\operatorname{diag}\left[\exp \left(i \gamma_{s} h / 2\right)\right]$. These phase-adjusted fields satisfy relationships analogous to Eqs. (22) and (23). Specifically, it follows immediately from Eqs. (22), (23), and (27) that

$$
\begin{aligned}
& \boldsymbol{F}_{D}^{-}=\tilde{\mathbf{T}}_{a} \boldsymbol{F}_{I}^{-}+\tilde{\mathbf{R}}_{b} \boldsymbol{F}_{I}^{+}+\mathbf{P} \mathbf{Q}^{-} \mathbf{Q}, \\
& \boldsymbol{F}_{D}^{+}=\tilde{\mathbf{R}}_{a} \boldsymbol{F}_{I}^{-}+\tilde{\mathbf{T}}_{b} \boldsymbol{F}_{I}^{+}+\mathbf{P} \mathbf{Q}^{+} \mathbf{Q},
\end{aligned}
$$

where $\tilde{\mathbf{R}}=$ PRP and $\tilde{\mathbf{T}}=\mathbf{P T P}$ are the corresponding reflection and transmission matrices. The phase origins of the fields $\boldsymbol{f}^{ \pm}$and $\boldsymbol{F}^{ \pm}$are depicted in Fig. 3, along with the scattering matrices associated with each surface of the waveguide layer.

In a partial or complete bandgap, the semi-infinite cladding immediately above and below the waveguide layer acts as a mirror. For such frequencies, it is possible to characterize the reflection from the cladding using another reflection matrix, $\mathbf{R}_{\infty}$, which is found from a Bloch mode analysis of the bulk woodpile. Obtaining the Bloch modes, in turn, only requires knowledge of the grating scattering matrices [24]. In order to induce a complete bandgap, consecutive pairs of gratings must be interleaved (the stacking unit comprises four layers rather than two). Consequently, the reflection matrix for the cladding below the waveguide layer $\left(\mathbf{R}_{\infty}\right)$ is different from the reflection matrix for the cladding above $\left(\mathbf{R}_{\infty}^{\prime}\right)$. The fields reflected back by the cladding are simply

$$
\boldsymbol{F}_{I}^{+}=\mathbf{R}_{\infty} \boldsymbol{F}_{D}^{-}, \quad \boldsymbol{F}_{I}^{-}=\mathbf{R}_{\infty}^{\prime} \boldsymbol{F}_{D}^{+}
$$

Equations (29)-(31) are then solved to find the incoming fields, which are found to be

$$
\boldsymbol{F}_{I}^{ \pm}=\mathbf{D}^{ \pm} \mathbf{Q}
$$

where

$$
\mathbf{D}^{ \pm}=\left(\mathbf{I}-\mathbf{G}^{\mp} \tilde{\mathbf{T}} \mathbf{G}^{ \pm}\right)^{-1}\left(\mathbf{G}^{\mp} \tilde{\mathbf{T}} \mathbf{G}^{ \pm} \mathbf{Q}^{ \pm}+\mathbf{G}^{\mp} \mathbf{Q}^{\mp}\right),
$$

$\mathbf{G}^{-}=\left(\mathbf{I}-\mathbf{R}_{\infty} \tilde{\mathbf{R}}\right)^{-1} \mathbf{R}_{\infty}, \quad \mathbf{G}^{+}=\left(\mathbf{I}-\mathbf{R}_{\infty}^{\prime} \tilde{\mathbf{R}}\right)^{-1} \mathbf{R}_{\infty}^{\prime}$, and where we have made use of the fact that for up-down symmetric gratings $\tilde{\mathbf{R}}_{a}=\tilde{\mathbf{R}}_{b} \stackrel{\text { def }}{=} \tilde{\mathbf{R}}$ and $\tilde{\mathbf{T}}_{a}=\tilde{\mathbf{T}}_{b} \stackrel{\text { def }}{=} \tilde{\mathbf{T}}$. Equations (24), (27), and (32) allow $\mathbf{B}$ to be expressed in the form

$$
\mathbf{B}=\mathbf{Z} \mathbf{Q},
$$

with

$$
\mathbf{Z}=\left(\mathbf{Y}^{-} \mathbf{P D}^{-}+\mathbf{Y}^{+} \mathbf{P D}^{+}+\mathbf{Y}^{f s}\right) \mathbf{Q} .
$$

Equations (25) and (34) completely determine the fictitious source coefficients, which are the solutions of

$$
(\mathbf{Z}-\mathbf{H}) \mathbf{Q}=\mathbf{0} .
$$


Suppose that the cylinders of the waveguide layer are aligned parallel to the $x$ axis, hence $k_{y}$ and $k_{x}$ are the components of $\mathbf{k}$ that are associated with the axis of the waveguide layer and with the direction parallel to the cylinders of this layer, respectively, as in Fig. $2(\mathrm{~b})$, i.e., $k_{x} \equiv \alpha_{0}$ and $k_{y} \equiv \beta_{0}$. When an average of the fields of the entire woodpile is taken with respect to $k_{y}$, where $k_{y}$ lies in the first BZ, the FSS multipole field expansions for the waveguide layer [Eqs. (3) and (13)] remain valid (as $k_{\perp}$ does not depend on $k_{y}$ ), except with the averaged coefficients $\left\langle A_{n}^{j, V}\right\rangle,\left\langle B_{n}^{j, V}\right\rangle,\left\langle C_{n}^{j, V}\right\rangle$, and $\left\langle Q_{n}^{j, V}\right\rangle$ used in place of $A_{n}^{j, V}, B_{n}^{j, V}, C_{n}^{j, V}$, and $Q_{n}^{j, V}$, where the averaging operator \langle\rangle is defined as

$$
\langle\cdot\rangle=\frac{d}{2 \pi} \int_{-\frac{\pi}{d}}^{\frac{\pi}{d}} \cdot \mathrm{d} \beta_{0} .
$$

The coefficients $Q_{n}^{0, V}$ [i.e., of the $j=0$ cylinder, see Eq. (13)] are chosen to be constant with respect to $k_{y}$. This constraint, along with the quasiperiodicity requirement, ensures that all fictitious sources in cylinders $j \neq 0$ disappear as a result of the averaging procedure, while the source in cylinder $j=0$ is unaffected, because

$$
\left\langle Q_{n}^{j, V}\right\rangle=Q_{n}^{0, V}\left\langle e^{i \beta_{0} j d}\right\rangle=\left\{\begin{array}{ll}
Q_{n}^{0, V}, & \text { for } j=0 \\
0, & \text { for } j \neq 0
\end{array},\right.
$$

and hence

$$
\langle\mathbf{Q}\rangle=\mathbf{Q},
$$

see Eq. (26). Consequently, $\mathbf{Q}$ can be used to control the outgoing fields, $\left\langle B_{n}^{0, V}\right\rangle$, or, equivalently, $\langle\mathbf{B}\rangle$, emanating from only the $j=0$ cylinder. It follows immediately from Eq. (25) that

$$
\langle\mathbf{B}\rangle=\langle\mathbf{H Q}\rangle=\mathbf{H Q},
$$

which relies on the fact that the boundary conditions do not depend on $k_{y}$, and so $\mathbf{H}=\langle\mathbf{H}\rangle$. Furthermore, applying the averaging operator to Eq. (34) yields

$$
\langle\mathbf{B}\rangle=\langle\mathbf{Z Q}\rangle=\langle\mathbf{Z}\rangle \mathbf{Q},
$$

hence, the choice of $\mathbf{Q}$ that produces the mimicked fields, $\langle\mathbf{B}\rangle$, is given by

$$
\left\langle\mathbf{Z}\left(k, k_{x}\right)-\mathbf{H}\left(k, k_{x}\right)\right\rangle \mathbf{Q}=\mathbf{0} ;
$$

see Equation (36). Evidently, searching for the defect modes of the linear waveguide amounts to determining the values of $k$ and $k_{x}$ for which Eq. (37) has nontrivial solutions, i.e., the values for which the coefficient matrix $\langle\mathbf{Z}-\mathbf{H}\rangle$ is singular (this can be ascertained, for example, by examining the determinant or the singular values).

\section{IMPLEMENTATION AND VERIFICATION}

In this section we discuss several considerations that are of practical significance. In addition, we use our supercell formulation [13] to verify our FSS code, and we also investigate the rate of convergence of the FSS method. The most important aspect of any FSS implementation is the discretization of the integral in Eq. (37). For simplicity, we used the trapezoidal rule to carry out the integration. However, as an artifact of using the trapezoidal rule, fictitious sources $\pm \mathbf{Q}$ are left in every $N$ unit cells, where $N$ is the number of subintervals used for the numerical integration over the $k_{y}$ direction of the $\mathrm{BZ}$, thereby imposing a supercell spanning $N$ cylinders onto the defect layer (this artifact can be avoided by using a different integration rule, e.g., Gaussian quadrature). Consequently, $N$ must be large enough to ensure that the amount of coupling between the fields of adjacent supercells is negligible. Further, when $N$ is even, the sign of $\mathbf{Q}$ is positive, which results in a periodic defect that repeats every $N$ cylinders, i.e., a CROW. This behavior provides a means for testing the correctness of our implementation because this type of structure can be modeled directly, albeit much less efficiently, using our supercell formulation [13]. Using Eqs. (20), (21), and (31), it is straightforward to demonstrate that the outgoing fields, $\boldsymbol{F}_{D}^{ \pm}$, incident on the waveguide layer satisfy

$$
\left(\mathbf{I}-\mathbf{E}^{\mp} \mathbf{E}^{ \pm}\right) \boldsymbol{F}_{D}^{ \pm}=0
$$

with

$$
\begin{aligned}
& \mathbf{E}^{-}=\left(\mathbf{I}-\tilde{\mathbf{R}}_{a} \mathbf{R}_{\infty}^{\prime}\right)^{-1} \tilde{\mathbf{T}}_{b} \mathbf{R}_{\infty}, \\
& \mathbf{E}^{+}=\left(\mathbf{I}-\tilde{\mathbf{R}}_{b} \mathbf{R}_{\infty}\right)^{-1} \tilde{\mathbf{T}}_{a} \mathbf{R}_{\infty}^{\prime},
\end{aligned}
$$

and where $\tilde{\mathbf{R}}$ and $\tilde{\mathbf{T}}$ are now the scattering matrices corresponding to a grating whose every $N_{s}$ th cylinder has been altered. As usual, one searches for values of $k$ and $k_{x}$ for which nontrivial solutions exist ( $k_{y}$ is redundant in this case). The equivalence of Eq. (38), i.e., the supercell method, and the FSS method using the trapezoidal rule can be seen in the results shown in Fig. 4 for the supercell method with $N_{s}=4$ and for the FSS method with $N=4$. Note that computing $\mathbf{R}_{\infty}$ for larger values of $N_{s}$ is prohibitively costly; thus, Eq. (38) is not suitable for locating the modes of a linear waveguide.

The overall running time of the FSS method is determined by the cost of computing $\langle\mathbf{Z}-\mathbf{H}\rangle$, which in turn is determined largely by the cost associated with constructing $\mathbf{R}_{\infty}$ and $\mathbf{R}_{\infty}^{\prime}$ for each mesh point of the numerical integration rule. Thus, the overall running time of the FSS method is linearly proportional to the number of mesh points. Hence, if the trapezoidal rule is used, then the overall running time of the FSS method is $O(N)$. This cost can be halved by observing that for a linear

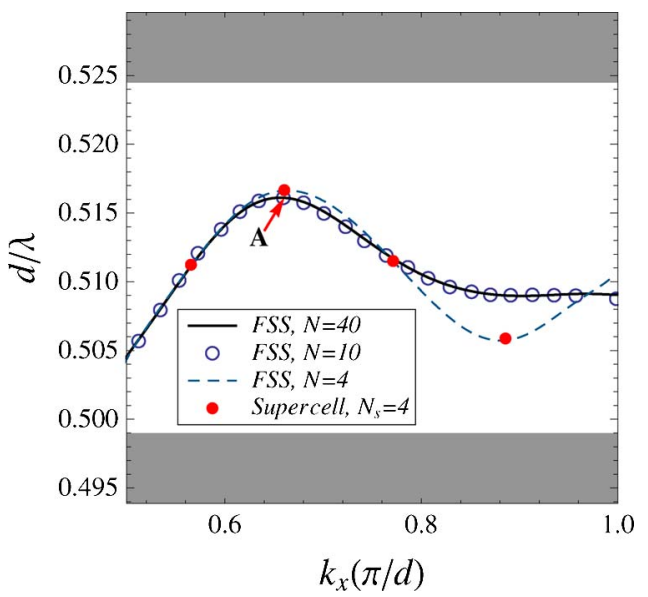

Fig. 4. (Color online) Comparison of the dispersion curves, computed using the FSS method with $N=4,10$, and 40 integration intervals, for the linear waveguide. The radius of the defect rod is $0.075 d$ (i.e., half that of the cladding rods). The frequency at the point labeled A, which corresponds to $k_{x}=2.075 / d$, is listed in Table 1 for different truncation parameters and for different values of $N$. Mode frequencies computed using Eq. (38) with a supercell containing $N_{s}=4$ cylinders are shown for comparison with the $N=4$ curve computed using the FSS method. Note that if the trapezoidal rule is used to carry out the integration (as it is here), then the FSS method is equivalent to the supercell method when $N_{s}=N$. 
waveguide, these reflection matrices are related via a similarity transform

$$
\mathbf{R}_{\infty}^{\prime}=\mathbf{S R}_{\infty} \mathbf{S}^{-1},
$$

where the similarity transform displaces the rods of the upper cladding by a distance $d / 2$ (or more generally by an amount $\delta$ ) in the direction parallel to the defect rod. That is, $\mathbf{S}$ is a diagonal matrix of the form

$$
\mathbf{S}=\left[\begin{array}{ll}
\boldsymbol{S} & \mathbf{0} \\
\mathbf{0} & \boldsymbol{S}
\end{array}\right],
$$

with $\boldsymbol{S}=\operatorname{diag}\left[\exp \left(-i \eta_{s} \delta\right)\right]$, so that if $(p, q)=\sigma^{-1}(s)$, then $\eta_{s \perp}=$ $\alpha_{p}$ if the defect runs parallel to the $x$ axis and $\eta_{s}=\beta_{q}$ otherwise.

Many of the matrices appearing in the preceding theory are sparse, and so the matrix algebra routines can be optimized to take advantage of this. In particular, the boundary condition matrices used to construct $\mathbf{H}$ are sparse and particularly simple in structure, making $\mathbf{H}$ inexpensive to compute. Thus, once $\langle\mathbf{Z}\rangle$ has been computed, $\langle\mathbf{Z}-\mathbf{H}\rangle \mathbf{Q}=\mathbf{0}$ can be solved for very little additional cost. Because the material parameters of the defect rod are completely encapsulated in $\mathbf{H}$, the frequencies of the defect modes can be determined quickly for many different values of these parameters (however, the positions of the defects are fixed).

The dispersion curves for the linear waveguide are presented in the next section. These were computed with the FSS method, where the integration step was carried out using the trapezoidal rule with $N=40$ integration intervals and where the diffraction orders and multipole orders were truncated to $p_{\max }=5$ and $n_{\max }=5$, respectively, i.e., so that $-p_{\max } \leq p, q \leq p_{\max }$ and $-n_{\max } \leq n \leq n_{\max }$, with $n$ as defined in Eq. (3). Table 1 shows how the frequency of the mode in Fig. $\underline{4}$ varies for different choices of $p_{\max }, n_{\max }$, and $N$, where the Bloch vector $k_{x}=2.075 / d$ is fixed (i.e., near the turning point, labeled A in Fig. 4). For $p_{\max }=5, n_{\max }=5$, and $N=40$, the error is less than $1 \%$ of the width of the complete bandgap, which spans the normalized frequencies $0.4990 \leq d / \lambda \leq 0.5245$ (the parameters of the woodpile are given in the next section).

\section{RESULTS}

For the following, the radii and refractive indices of the cylinders of the unperturbed woodpile are $r=0.15 d$ and $n_{c}=2.68$ (chalcogenide glass), while the background refractive index is $n_{b}=1$. The interlayer spacing is $h=0.3002 d$, so that the cylinders in adjacent layers are almost touching, and each pair of

Table 1. Convergence of the (Normalized) Frequencies, $d / \lambda$, Near Point A of Fig. 4, with $k_{x}=2.075 / d$ Being Fixed

\begin{tabular}{cccc}
\hline$d / \lambda$ & $p_{\max }$ & $n_{\max }$ & $N$ \\
\hline 0.516643 & 3 & 9 & 80 \\
0.516275 & 4 & 9 & 80 \\
0.516161 & 5 & 5 & 40 \\
0.516122 & 5 & 7 & 40 \\
0.516120 & 5 & 9 & 40 \\
0.516115 & 5 & 9 & 80 \\
0.516059 & 6 & 7 & 80 \\
0.516035 & 7 & 7 & 80 \\
\hline
\end{tabular}

consecutive layers is offset laterally by a distance $d / 2$ in both the $x$ and $y$ directions with respect to pairs immediately above and below it. The cladding has a complete bandgap that lies above the light line $k n_{b}=k_{x}$, with $0 \leq k_{x} \leq \pi / d$. Further, the complete gap spans the normalized frequencies $0.4990 \leq$ $d / \lambda \leq 0.5245$. For these frequencies, the confinement of any mode propagating along the waveguide is due entirely to the bandgap. The BZ integration was carried out using the trapezoidal rule. While this imposes an artificial supercell onto the defect layer (as discussed earlier), the size of the supercell can be increased at little additional computational expense.

In the first instance, a linear waveguide was created by decreasing the radius, $r_{d}$, of a single cylinder of an otherwise uniform woodpile. Figure 4 shows part of the dispersion curve of one of the modes for $r_{d}=0.5 r$, where the curve was computed using the FSS method with 40 integration intervals. Figure 4 also shows the dispersion curves as computed using $N=4$ and $N=10$. Note that using $N=10$ effectively models a grating supercell containing only 10 cylinders yet there is still good agreement with the values obtained using $N=40$ intervals, indicating that the lateral confinement of the modes is quite strong.

Figure $\underline{5}$ shows the dispersion curves for each mode and for several values of $r_{d}$, where the curves were computed using the FSS method with $N=40$ integration subintervals. For each value of $r_{d}$ there is a pair of defect modes inside the bandgap. As is to be expected, decreasing $r_{d}$, and hence removing some dielectric, has the effect of increasing the frequency of the defect modes. The behavior of the modes for any given $r_{d}$ is quite different from that of typical waveguiding modes of planar PC waveguides in that one of the two modes displays unusual quartic dispersion. Furthermore, the two modes become degenerate precisely at the BZ edge (i.e., at $k_{x} d / \pi=1.0$ ). This degeneracy has previously been observed in woodpile waveguides structures $[\underline{11}, \underline{12}, \underline{18}-20]$, and it can be attributed to the fact that the waveguide possesses glidereflection symmetry in the waveguiding direction [27], i.e., the waveguide is invariant under the transformation $(z \rightarrow-z)$, $(x \rightarrow x+d / 2)$. Further, the frequency of the quartic mode decreases more rapidly than that of the quadratic mode as $r_{d}$ increases. Thus, because the frequencies of these two

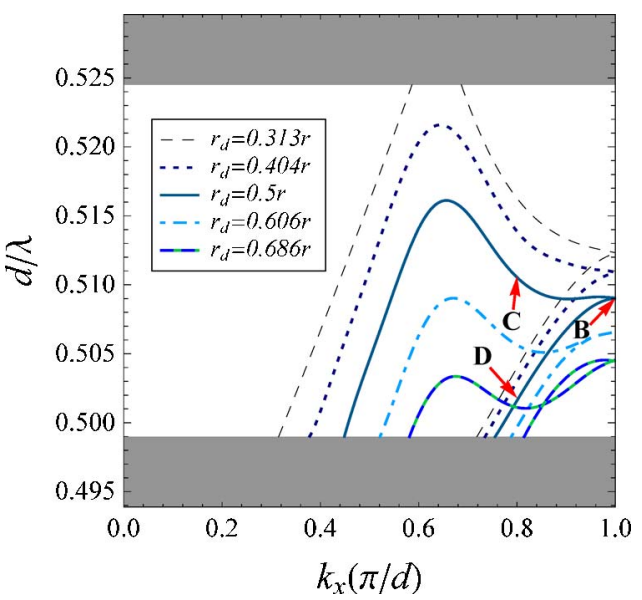

Fig. 5. (Color online) Dispersion curves for the linear waveguide for different values of $r_{d}$, which specifies the radius of the defect rod. Here, $r$ is the radius of the cladding rods. Note that the complete bandgap of the cladding spans the normalized frequencies $0.4990 \leq d / \lambda \leq 0.5245$ 
modes are always the same at the edge of the BZ, the dispersion curve of the quartic mode can be made to cross that of the quadratic mode simply by making $r_{d}$ sufficiently large (specifically, by choosing $r_{d} / r \gtrsim 0.6$ ). We found that defect modes appeared as soon $r_{d} \neq r$, and that for shallow defects $\left(r_{d} / r \gtrsim 0.95\right)$, the corresponding dispersion curves are flat and very close to the low-frequency edge of the bandgap. Note that for such small perturbations, the defect modes necessarily become highly extended, and, thus, it is extremely difficult, if not impossible, to use a supercell approach to model a shallow defect, whereas the FSS method is well suited for the task.

Furthermore, for $r_{d}=0.5 r$, there is a region near the edge of the $\mathrm{BZ}\left(0.85 \lesssim k_{x} d / \pi \leq 1.0\right.$, which corresponds to about $15 \%$ of the BZ) over which the frequency of the higher branch is almost constant, $d / \lambda \approx 0.5090$, and quite close to the middle of the bandgap. This region meets the slow-light criterion $v_{x} / c \ll 1$, and, for example, can thus be exploited to enhance nonlinear optical processes, because chalcogenide glass exhibits a strong nonlinear response [28]. The electric and magnetic energy densities for point $\overline{\mathrm{B}}$ of Fig. 5 are shown in Fig. 6 for planes $x / d=0.0$ and $x / d=0.25$, where $x$ is the direction parallel to the defect rod. Note that point $\mathrm{B}$ corresponds to $k_{x} d / \pi=1.0$ and $d / \lambda \approx 0.5091$, and it lies inside the slow-light region for $r_{d}=0.5 r$. For both the lateral and stacking directions, there is strong localization of the electric and magnetic fields. In particular, the electric field for $x / d=$ 0.25 is similar to that of a waveguiding mode of a photoniccrystal fiber. The energy flux in the waveguiding direction,

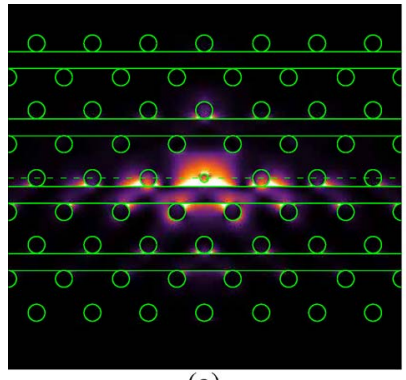

(a)

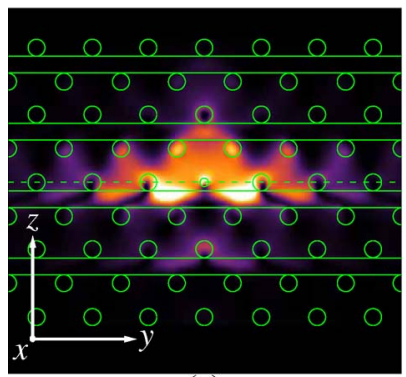

(c)

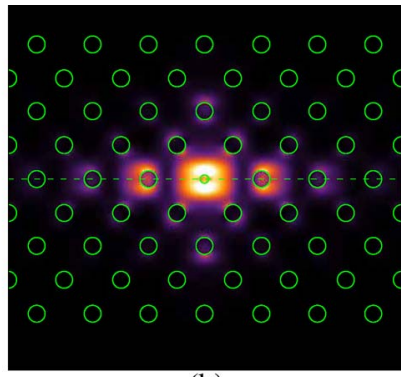

(b)

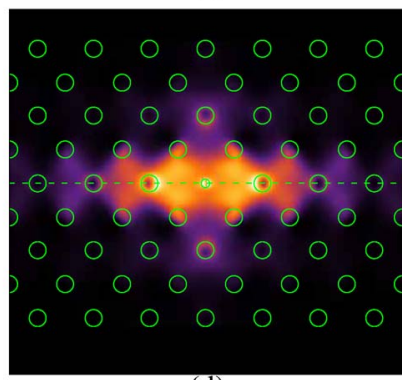

(d)

$$
0.5 \geq 1.0
$$

Arb. units

Fig. 6. (Color online) Plots of the energy density, $\varepsilon\|\boldsymbol{E}\|^{2}$, of the electric field [(a) and (b)], and of the energy density, $\mu\|\boldsymbol{H}\|^{2}$, of the magnetic field [(c) and (d)] for the linear waveguide for the "slow-light" point, i.e., point $\mathrm{B}\left(k_{x} d / \pi=1.0, d / \lambda \approx 0.5091\right)$, indicated in Fig. $\underline{5}$, where $\varepsilon(y, z)$ and $\mu(y, z)$ are the relative permittivity and relative permeability. The view looks down the defect rod-the radius of which is $r_{d}=0.5 r$. Plots (a) and (c) are for the plane $x / d=0.0$, where $x$ is the direction parallel to the defect rod. Plots (b) and (d) are for the plane $x / d=0.25$. i.e., $S_{x}$, where $\mathbf{S}=\operatorname{Re}\left(\mathbf{E} \times \mathbf{H}^{*}\right) / 2$ is the Poynting vector, is shown in Fig. 7 for planes $x / d=0.0$ and $x / d=0.25$, with $k_{x}$ fixed at point $\mathrm{B}$ as before. The magnitude of $S_{x}$ is greatest in the vicinity of the defect rod; however, in this region $S_{x}$ assumes positive values as often as it does negative values; thus the net flux is approximately zero, leading to a low group velocity $\left(v_{x}\right)$ along the waveguide. Figure $\underline{7}$ also shows $S_{x}$ at point $\mathrm{C}\left(d / \lambda=0.5105, k_{x}=0.8 \pi / d\right)$ indicated in Fig. 5 . This point lies on the same dispersion curve as point B (i.e., on the high-frequency branch of the curve for $r_{d}=0.5 r$ ); however, unlike point $\mathrm{B}$, point $\mathrm{C}$ does not lie inside the slow-light region, and it can be seen that $S_{x}$ is largely negative when $x / d=0.25$, resulting in a relatively large $v_{x}$. Also shown in Fig. $\underline{7}$ are plots of $S_{x}$ for point D $\left(d / \lambda=0.5102, k_{x}=0.8 \pi / d\right)$ of Fig. 5. This point lies on the low-frequency branch of the quadratic dispersion curve for $r_{d}=0.5 r$, and it can be seen for this mode that $S_{x}$ is mostly positive, and, while much of the energy is transported along the defect rod, there is considerable flux along the rods on either side of the defect. Further, the flux is only appreciable in the waveguide layer and in the layers immediately above and below it.

We have also modeled a waveguide that was created by decreasing only the refractive index, $n_{d}$, of a single rod, while
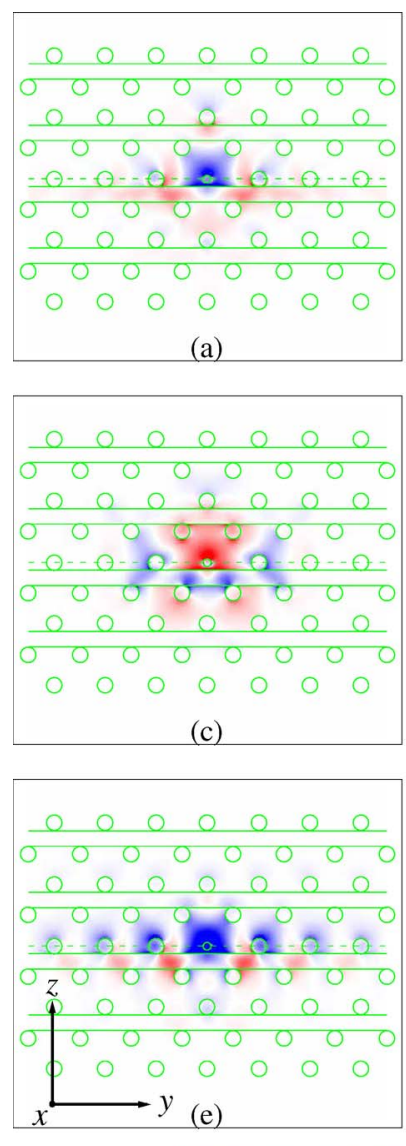

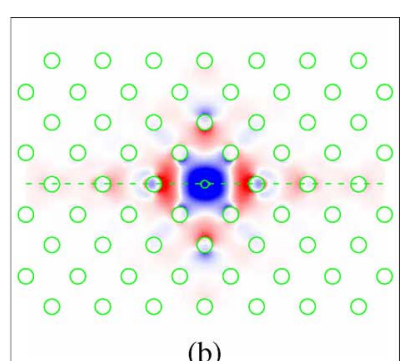

(b)

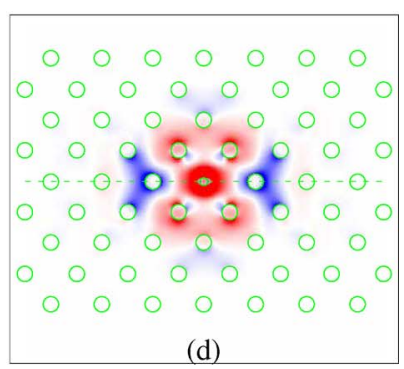

(d)

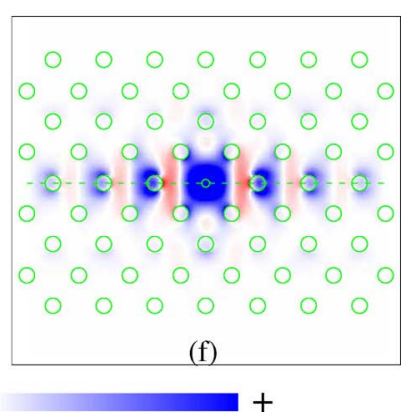

Fig. 7. (Color online) Plot of $\operatorname{Re}\left(S_{x}\right)$ for points B [parts (a) and (b)] (Media 1), C [parts (c) and (d)] (Media 2), and D [parts (e) and (f)] (Media 3 ) of Fig. 5 , where $S_{x}$ is the $x$ component of the Poynting vector. The view looks down the defect rod, the radius of which is $r_{d}=0.5 r$. Plots (a), (c), and (e) are for the plane $x / d=0.0$, where $x$ is the direction parallel to the defect rod. Plots (b), (d), and (f) are for the plane $x / d=0.25$. Animations that show how $\operatorname{Re}\left(S_{x}\right)$ varies as $x$ moves along the defect rod are available online. 


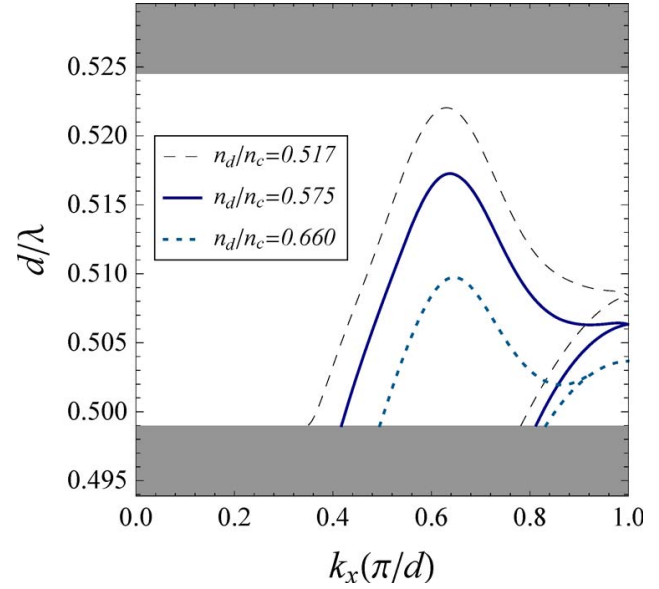

Fig. 8. (Color online) Dispersion curves for the linear waveguide for different values of $n_{d}$, which specifies the refractive index of the defect rod. The refractive index of the cladding rods is $n_{c}=$ 2.68 (chalcogenide glass).

leaving the radius of the rod unchanged. The dispersion curves for this structure are shown in Fig. 8. Decreasing $n_{d}$, so that $n_{b}<n_{d}<n_{c}$, has an effect similar to reducing the radius of the defect, with lower $n_{d}$ resulting in a higher frequency (both types of defects are "acceptor-type" defects, which are created by removing some dielectric; see, e.g., [20]). Once again, we observe the appearance of a slow-light mode at the edge of the BZ. In particular, the breadth of the slow-light region is maximized for $n_{d} / n_{c} \approx 0.575$. For $0.90 \lesssim$ $n_{d} / n_{c}<1.0$, i.e., for reasonably small perturbations, the defect modes have frequencies very close to the low-frequency edge of the bandgap, as was the case when $r_{d} \approx r$. The degeneracy of the defect modes at the edge of the BZ (i.e., at $\left.k_{x}=\pi / d\right)$ is again evident because the defect preserves the glide-reflection symmetry of the waveguide.

\section{CONCLUSION}

We have extended the FSS method so that it can be used to model linear defects in woodpiles. Our results indicate that creating a defect by decreasing the filling fraction of a single rod always results in defect states, even when the perturbation is only about $1 \%$. Further, the symmetry of the woodpile structure means that the waveguiding modes are always degenerate at the edge of the BZ. The efficiency of the method was such that we were able to vary the parameters of the defect rod in a continuous manner. It was found that choosing the radius of the defect to be half that of the cladding rods resulted in a waveguide having very flat dispersion over $\sim 15 \%$ of BZ. In PC waveguides, scattering losses due to extrinsic factors appear to scale inversely with group velocity [8], and, consequently, fabricating a PC waveguide that is capable of operating in the slow-light regime is problematic. Extrinsic scattering losses in planar PC waveguides arise from backscattering and out-of-plane scattering, where in-plane scattering losses can be mitigated by increasing the number of layers of the periodic pattern. For the waveguide considered here, the complete bandgap of the woodpile cladding can be used to eliminate out-of-plane scattering, making this a compelling geometry for slow-light waveguides, provided that the amount of backscattering is only moderate. The importance of backscattering in slow-light woodpile waveguides remains an open question; however, quantifying the amount of backscattering is beyond the scope of this paper.

\section{ACKNOWLEDGMENTS}

This work was supported by the Australian Research Council under its Centres of Excellence Program. C. G. Poulton acknowledges the support of a startup grant awarded by the University of Technology, Sydney. The results in this paper were obtained using the supercomputers at the NCI National Facility. The authors thank S. Mahmoodian for his helpful comments.

\section{APPENDIX A: EXPRESSIONS FOR THE FICTITIOUS SOURCE SUPERPOSITION BOUNDARY CONDITION MATRIX}

The matrix $\mathcal{N}$ encapsulating the boundary conditions for the fictitious sources [see Eq. (14)] is a 2 by 2 block matrix, where the superscripts $E$ and $H$ are used to label the blocks so that

$$
\boldsymbol{N}=\left[\begin{array}{ll}
\mathbf{N}^{E E} & \mathbf{N}^{E H} \\
\mathbf{N}^{H E} & \mathbf{N}^{H H}
\end{array}\right] .
$$

Furthermore, each block is diagonal, so we may use $n$ to index the elements of a given block. The label "I" denotes quantities that depend on the properties of the background region, while "II" denotes quantities that depend on the material properties of the cylinder. Let $r$ be the radius of the cylinder, and let $\varepsilon_{j}$ and $\mu_{j}$ be the permittivity and permeability, respectively, of region $j$ (i.e., $j=\mathrm{I}$ or $j=$ II). If $k_{\perp j}$ is the transverse wavenumber inside region $j$, so that $k_{\perp_{j}}^{2}=k^{2} \varepsilon_{j} \mu_{j}-\alpha_{p}^{2}$, then the diagonal entries of the blocks are

$$
N_{n}^{E E}=\eta_{3}(\mathrm{II}) J_{2} H / \Delta,
$$

$$
N_{n}^{H H}=\eta_{2}(\mathrm{II}) J_{3} H / \Delta,
$$

$$
N_{n}^{E H}=\eta_{2}(\mathrm{II})\left[\eta_{1}(\mathrm{I})-\eta_{1}(\mathrm{II})\right] H / \Delta,
$$

$$
N_{n}^{H E}=\eta_{3}(\mathrm{II})\left[\eta_{1}(\mathrm{I})-\eta_{1}(\mathrm{II})\right] H / \Delta,
$$

where $\quad \eta_{1}(j)=-\alpha_{p} n /\left[\left(k_{\perp j}\right)^{2} r\right], \quad \eta_{2}(j)=-i k \mu_{j} / k_{\perp j}, \quad \eta_{3}(j)=$ $i k \varepsilon_{j} / k_{\perp j}$,

$$
\begin{gathered}
\Delta=J_{n}\left(k_{\perp \mathrm{I}} r\right)^{2}\left\{\left[\eta_{1}(\mathrm{I})-\eta_{1}(\mathrm{II})\right]^{2}-J_{2} J_{3}\right\}, \\
J_{j}=\eta_{j}(\mathrm{I})\left\{\frac{J_{n}^{\prime}\left(k_{\perp \mathrm{I}} r\right)}{J_{n}\left(k_{\perp \mathrm{I}} r\right)}-\frac{\eta_{j}(\mathrm{II}) J_{n}^{\prime}\left(k_{\perp \mathrm{II}} r\right)}{\eta_{j}(\mathrm{I}) J_{n}\left(k_{\perp \mathrm{II}} r\right)}\right\}, \\
H=J_{n}\left(k_{\perp \mathrm{I}} r\right) H_{n}\left(k_{\perp \mathrm{II}} r\right) \times\left\{\frac{H_{n}^{\prime}\left(k_{\perp \mathrm{II}} r\right)}{H_{n}\left(k_{\perp \mathrm{II}} r\right)}-\frac{J_{n}^{\prime}\left(k_{\perp \mathrm{II}} r\right)}{J_{n}\left(k_{\perp \mathrm{II}} r\right)}\right\} .
\end{gathered}
$$

\section{APPENDIX B: GRATING SCATTERING MATRICES}

The scattering matrices appearing in Eqs. (11) and (12) are partitioned into blocks appropriately, so that 


$$
\mathcal{R}_{p}=\left[\begin{array}{ll}
\mathcal{R}^{\mathrm{TE}, \mathrm{TE}} & \mathcal{R}^{\mathrm{TE}, \mathrm{TM}} \\
\mathcal{R}^{\mathrm{TM}, \mathrm{TE}} & \mathcal{R}^{\mathrm{TM}, \mathrm{TM}}
\end{array}\right]
$$

with $\mathcal{T}_{p}$ being similarly defined; hence,

$$
\begin{aligned}
f_{D,\left(p, q_{o}\right)}^{V+}= & \sum_{q_{i}} \mathcal{R}_{q_{o}}^{V, \mathrm{TE}} f_{I,\left(p, q_{i}\right)}^{\mathrm{TE}-}+\mathcal{R}_{q_{o} q_{i}}^{V, \mathrm{TM}} f_{I,\left(p, q_{i}\right)}^{\mathrm{TM}-}+\mathcal{T}_{q_{o} q_{i}}^{V, \mathrm{TE}} f_{I,\left(p, q_{i}\right)}^{\mathrm{TE}+} \\
& +\mathcal{T}_{q_{o} q_{i}}^{V, \mathrm{TM}} f_{I,\left(p, q_{i}\right)}^{\mathrm{TM}+} .
\end{aligned}
$$

Here, $V$ specifies the polarization of the output field (i.e., either TE or TM), $\left(p, q_{o}\right)$ is the output channel, with $p$ fixed (as usual) and $q_{o}$ arbitrary, and $\left(p, q_{i}\right)$ specifies an input channel and the summation is taken over the set of all the input channels that have an $x$ dependency of $\exp \left(i \alpha_{p} x\right)$.

Similarly, $\mathbf{R}$ (and also T) appearing in Eqs. (20) and (21) is partitioned in the form

$$
\mathbf{R}=\left[\begin{array}{ll}
\mathbf{R}^{\mathrm{TE}, \mathrm{TE}} & \mathbf{R}^{\mathrm{TE}, \mathrm{TM}} \\
\mathbf{R}^{\mathrm{TM}, \mathrm{TE}} & \mathbf{R}^{\mathrm{TM}, \mathrm{TM}}
\end{array}\right],
$$

which allows each coefficient of the diffracted fields to be expressed as

$$
f_{D, s_{o}}^{V+}=\sum_{s_{i}} \mathbf{R}_{s_{o}}^{V, s_{i}} f_{I, s_{i}}^{\mathrm{TE}-}+\mathbf{R}_{s_{o} s_{i}}^{V, \mathrm{TM}} f_{I, s_{i}}^{\mathrm{TM}-}+\mathbf{T}_{s_{o} s_{i}}^{V, \mathrm{TE}} f_{I, s_{i}}^{\mathrm{TE}+}+\mathbf{T}_{s_{o} s_{i}}^{V, \mathrm{TM}} f_{I, s_{i}}^{\mathrm{TM}+}
$$

where again $V=\mathrm{TE}$ or TM, $s_{o}=\boldsymbol{\sigma}\left(p_{o}, q_{o}\right)$ is the output channel; however, now $p_{o}$ also assumes arbitrary values, $s_{i}=$ $\boldsymbol{\sigma}\left(p_{i}, q_{i}\right)$ is the input channel, and the summation is taken over all input channels. Note that the choice of which bijection to use (i.e., either $\boldsymbol{\sigma}$ or $\boldsymbol{\sigma}^{\prime}$ ) depends on the orientation of the rods. The elements of $\mathbf{R}^{\mathrm{TE}, \mathrm{TE}}$, for example, are taken directly from each $\mathcal{R}_{p_{o}}^{\mathrm{TE}, \mathrm{TE}}$ of Eq. (B1) according to (the subscript $p_{o}$ is used to emphasize that there is a different $\mathcal{R}^{\mathrm{TE}, \mathrm{TE}}$ for each value of $\left.p_{o}\right)$

$$
\mathbf{R}^{\mathrm{TE}, \mathrm{TE}}\left[s_{o}, s_{i}\right]= \begin{cases}\mathcal{R}_{p_{o}}^{\mathrm{TE}, \mathrm{TE}}\left[q_{o}, q_{i}\right], & \text { for } p_{o}=p_{i} \\ 0, & \text { otherwise }\end{cases}
$$

\section{REFERENCES}

1. J. D. Joannopoulos, S. G. Johnson, J. N. Winn, and R. D. Meade, Photonic Crystals: Molding the Flow of Light (Princeton University, 2008).

2. H. S. Sözüer and J. P. Dowling, "Photonic band calculations for woodpile structures," J. Mod. Opt. 41, 231-239 (1994).

3. K. M. Ho, C. T. Chan, C. M. Soukoulis, R. Biswas, and M. Sigalas, "Photonic band gaps in three dimensions: new layer-by-layer periodic structures," Solid State Commun. 89, 413-416 (1994).

4. N. Yamamoto, S. Noda, and A. Chutinan, "Development of one period of a three-dimensional photonic crystal in the $510 \mu \mathrm{m}$ wavelength region by wafer fusion and laser beam diffraction pattern observation techniques," Jpn. J. Appl. Phys. 37, L1052-L1054 (1998).

5. J. G. Fleming, S. Y. Lin, I. El-Kady, R. Biswas, and K. M. Ho, "All-metallic three-dimensional photonic crystals with a large infrared bandgap," Nature 417, 52-55 (2002).

6. E. Nicoletti, G. Zhou, B. Jia, M. J. Ventura, D. Bulla, B. LutherDavies, and M. Gu, "Observation of multiple higher-order stopgaps from three-dimensional chalcogenide glass photonic crystals," Opt. Lett. 33, 2311-2313 (2008).
7. T. F. Krauss, "Why do we need slow light?" Nat. Photon. 2, 448450 (2008).

8. S. Hughes, L. Ramunno, J. F. Young, and J. E. Sipe, "Extrinsic optical scattering loss in photonic crystal waveguides: role of fabrication disorder and photon group velocity,” Phys. Rev. Lett. 94, 033903 (2005).

9. S. Johnson, M. Povinelli, M. Soljačić, A. Kralis, S. Jacobs, and J. Joannopoulos, "Roughness losses and volume-current methods in photonic-crystal waveguides," Appl. Phys. B 81, 283-293 (2005).

10. S. Ogawa, M. Imada, S. Yoshimoto, M. Okano, and S. Noda, "Control of light emission by 3D photonic crystals," Science 305, 227-229 (2004).

11. M. Imada, L. H. Lee, M. Okano, S. Kawashima, and S. Noda, "Development of three-dimensional photonic-crystal waveguides at optical-communication wavelengths," Appl. Phys. Lett. 88, 171107 (2006).

12. S. Kawashima, K. Ishizaki, and S. Noda, "Light propagation in three-dimensional photonic crystals," Opt. Express 18, 386-392 (2010).

13. D. J. Kan, A. A. Asatryan, C. G. Poulton, and L. C. Botten, "Multipole method for modeling linear defects in photonic woodpiles," J. Opt. Soc. Am. B 27, 246-258 (2010).

14. J. Chen, R. Hong, and J. Yang, "Analysis of planar defect structures in three-dimensional layer-by-layer photonic crystals," J. Appl. Phys. 104, 063111 (2008).

15. S. G. Johnson and J. D. Joannopoulos, "Block-iterative frequency-domain methods for Maxwell's equations in a plane wave basis," Opt. Express 8, 173-190 (2001).

16. A. Taflove and S. C. Hagness, Computational Electrodynamics: The Finite-Difference Time-Domain Method (Artech House, 2005).

17. A. Chutinan and S. Noda, "Highly confined waveguides and waveguide bends in three-dimensional photonic crystals," Appl. Phys. Lett. 75, 3739-3741 (1999).

18. M. Okano, S. Kako, and S. Noda, "Coupling between a pointdefect cavity and a line-defect waveguide in three-dimensiona photonic crystal," Phys. Rev. B 68, 235110 (2003).

19. S. Kawashima, L. H. Lee, M. Okano, M. Imada, and S. Noda, "Design of donor-type line-defect waveguides in three-dimensional photonic crystals," Opt. Express 13, 9774-9781 (2005).

20. S. Kawashima, M. Okano, M. Imada, and S. Noda, "Design of compound-defect waveguides in three-dimensional photonic crystals," Opt. Express 14, 6303-6307 (2006).

21. S. Wilcox, L. C. Botten, R. C. McPhedran, C. G. Poulton, and C. M. de Sterke, "Modeling of defect modes in photonic crystals using the fictitious source superposition method," Phys. Rev. E 71, 056606 (2005)

22. L. C. Botten, K. B. Dossou, S. Wilcox, R. C. McPhedran, C. M. de Sterke, N. A. Nicorovici, and A. A. Asatryan, "Highly accurate modelling of generalized defect modes in photonic crystals using the fictitious source superposition method," Int. J. Microw. Opt. Technol. 1, 133-145 (2006).

23. F. Zolla, R. Petit, and M. Cadilhac, "Electromagnetic theory of diffraction by a system of parallel rods: the method of fictitious sources," J. Opt. Soc. Am. A 11, 1087-1096 (1994).

24. G. H. Smith, L. C. Botten, R. C. McPhedran, and N. A. Nicorovici, "Cylinder gratings in conical incidence with applications to woodpile structures," Phys. Rev. E 67, 056620 (2003).

25. A. Figotin and V. Goren, "Resolvent method for computations of localized defect modes of H-polarization in two-dimensional photonic crystals," Phys. Rev. E 64, 056623 (2001).

26. L. C. Botten, N. A. Nicorovici, A. A. Asatryan, R. C. McPhedran, C. M. de Sterke, and P. A. Robinson, "Formulation for electromagnetic scattering and propagation through grating stacks of metallic and dielectric cylinders for photonic crystal calculations. part II. properties and implementation," J. Opt. Soc. Am. A 17, 2177-2109 (2000).

27. S. Ha, A. A. Sukhorukov, K. B. Dossou, L. C. Botten, A. V. Lavrinenko, D. N. Chigrin, and Y. S. Kivshar, "Dispersionless tunneling of slow light in antisymmetric photonic crystal couplers," Opt. Express 16, 1104-1114 (2008).

28. S. J. Madden, D. Choi, M. R. Lamont, V. G. Taeed, N. J. Baker, M. D. Pelusi, B. Luther-Davies, and B. J. Eggleton, "Chalcogenide glass photonic chips," Opt. Photon. News 19, 19-23 (2008). 